This item was submitted to Loughborough's Research Repository by the author.

Items in Figshare are protected by copyright, with all rights reserved, unless otherwise indicated.

\title{
Abrasion resistance of hydrophobic, anti-soiling coatings for solar cover glass
}

PLEASE CITE THE PUBLISHED VERSION

https://doi.org/10.1109/pvsc45281.2020.9300904

PUBLISHER

IEEE

VERSION

AM (Accepted Manuscript)

\section{PUBLISHER STATEMENT}

Personal use of this material is permitted. Permission from IEEE must be obtained for all other uses, in any current or future media, including reprinting/republishing this material for advertising or promotional purposes, creating new collective works, for resale or redistribution to servers or lists, or reuse of any copyrighted component of this work in other works.

\section{LICENCE}

\section{All Rights Reserved}

\section{REPOSITORY RECORD}

Bukhari, Farwah, Fabiana Lisco, Sona Ulicna, Kurt Barth, and Michael Walls. 2021. "Abrasion Resistance of Hydrophobic, Anti-soiling Coatings for Solar Cover Glass". Loughborough University. https://hdl.handle.net/2134/13603010.v1. 


\title{
Abrasion resistance of hydrophobic, anti-soiling coatings for solar cover glass
}

\author{
Farwah Bukhari ${ }^{1}$, Fabiana Lisco ${ }^{1}$, Soňa Uličná ${ }^{1}$, Kurt Barth ${ }^{2}$ and John M. Walls ${ }^{1}$ \\ ${ }^{1}$ CREST, Wolfson School of Mechanical, Electrical and Manufacturing Engineering, Loughborough University, Loughborough, \\ Leicestershire, LE11 3TU, UK \\ ${ }^{2}$ Colorado State University, Fort Collins, Colorado USA.
}

\begin{abstract}
Soiling is a serious problem for asset managers since it reduces the power output of solar modules and the costs of maintenance adversely affects the return on investment. Hydrophobic, anti-soiling coatings offer a potential solution to this problem. However, module cleaning can cause damage to the coatings through abrasion. Abrasion resistance tests have been carried out on candidate anti-soiling coatings with differing chemistry, cured and deposited at different conditions. In this work, we have studied the effects of linear abrasion and washability to assess the susceptibility of anti-soiling coatings to abrasion damage. Preliminary results indicate that great care must be taken over the choice of cleaning materials used. The results also confirm that rigorous laboratory testing of coatings is necessary before they are deployed at scale in the field for solar applications.
\end{abstract}

Keywords - photovoltaics, modules, cover glass, anti-soiling, abraders, cleaning methods, hydrophobic, coatings

\section{INTRODUCTION}

Solar asset managers have identified soiling of module cover glass as an important issue since attenuation of light is directly related to lower power output. The solar research community is making progress in understanding the nature of soiling and its dependence on geographical and climate conditions [1]. Strategies are now being developed to help mitigate the power loss caused by soiling and to reduce the associated costs of cleaning and maintenance. Textured glass, robotic and manual cleaning systems, self-cleaning and anti- soiling coatings are active areas of research being pursued to address the problem. Anti-soiling coatings for the cover glass hold out the promise of a low-maintenance solution to mitigate soiling. Both hydrophilic and hydrophobic coatings for PV application are under development. The promise of hydrophobic coatings has been demonstrated in outdoor testing although degradation of the coating chemistry remains a problem [1]. Laboratory testing such as UV and damp heat exposure has uncovered the degradation mechanisms responsible for coating degradation. However, outdoor exposure combines all the environmental stresses together and degradation occurs surprisingly quickly. Coating degradation manifests by both the water contact angle and the roll-off angle deterioration [2]. Even with an effective anti-soiling coating, cover glass surfaces may need to be cleaned. Cleaning may cause abrasion of the coating leading to removal or further degradation. This work is focused on the damage caused by abrasion to candidate hydrophobic, antisoiling coatings under development for the solar application.
Hydrophobic coatings are generally fluorinated polysiloxanes or other polymer based, with or without the addition of functionalised nanoparticles. Some are deposited on a hard underlayer to improve abrasion resistance. Abrasion can be caused by environmental factors since the cover glass can be exposed to dust, sand, and hail impact. These impacts can potentially remove areas of the coating if the coating is not mechanically durable. Abrasion can also be caused by handling during installation or during routine maintenance and by manually or automated methods of cleaning.

Abrasion is simulated in the laboratory using linear abrasion testing equipment with a range of abrasive pads with increasing hardness [3]. Washability testing can also be carried out to include the effects of water or solvents. The effects of sand impact requires custom built testing facilities [4] This range of tests can be used to assess the damage caused to coatings due to environmental factors or routine cleaning procedures.

Formal test protocols and standards have not yet been formulated for anti-soiling coatings for use on solar modules. Here we report on the use of linear abrasion to simulate natural and artificial abrasion mechanisms to determine how quickly the coatings lose their hydrophobicity. The abrasion tests were carried out on coatings that performed well in accordance IEC 61215-2 (MQT-10 and MQT-13) [5] for Damp Heat and UV exposure. 


\section{EXPERIMENTAL}

\section{A. Linear Abrasion Tests}

Tests were carried out on hydrophobic coatings deposited on $5 \mathrm{~cm} \times 5 \mathrm{~cm}$ glass substrates. A Taber Linear abrasion tester, model 5900 (Fig. 1a) was used and tests methods were adapted from BS EN 1096-2[6], ISO 9211-4 [7]. CS-10 and CS-8, felt pad and cheesecloth were the abraders used with $5 \mathrm{~N}$ load at 50 cycles/minute, with a total of 100 cycles. An intermediate check was performed after 50 cycles for all the tests. The coatings were applied to glass coupons by using dip, spray and wipe coating methods. The stroke length for all the cycles of abrasion was 30 $\mathrm{mm}$.

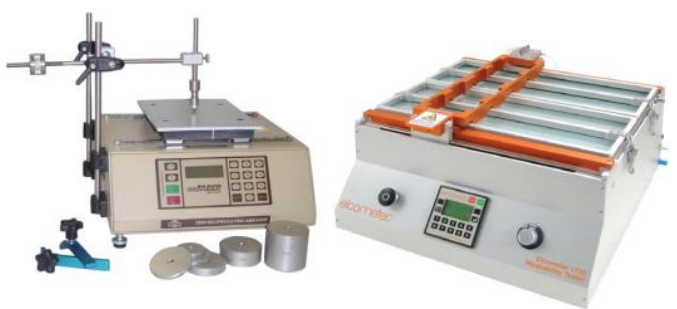

Fig. 1. a) Taber Linear abrader model 5900 and b) Elcometer1720, 4 Station

Any particulates collected on coupons after the abrasion tests were removed by blowing the surface with compressed air prior to inspection. Changes in hydrophobicity were assessed by rolloff angle (RoA) and water contact angle measurements (WCA). Scanning Electron Microscopic images obtained by JEOL 7800F FEGSEM were used to analyse the surface damage.

\section{B. Washability Tests}

The coatings were tested by wet scrubbing methods adapted from standards ISO 11998[8], ASTM D2486[9] and D4213[10]. An Elcometer 1720, 4 Station; Abrasion and Washability Tester (Fig. 1b) was employed, which is typically used to measure the durability of paints and varnishes. Washability tests were performed using 5 tools, Wild boar Brush(250g), Nylon Brush(454g), Sponge(508g), Sponge750g), Abrasive (135g), using DI water $(5 \mathrm{ml}-15 \mathrm{ml})$ as per standards mentioned above. Coatings were subjected to 200 cycles of abrasion at a stroke length of $300 \pm 5 \mathrm{~mm}$ was used at a rate of 37 cycles per minute to comply with the above-mentioned standards. The coated coupons were then analysed to assess the damage by measuring the change in WCA and RoA before and after the test. The use of water in these tests reduces the friction between the coupons and the washer tool. This is more representative of cleaning solar panels using water.

\section{RESULTS AND DISCUSSION}

\section{A. Linear Abrasion Tests}

The abrasion resistance of coatings applied by spraying, wiping and dipping were compared. The effect of abrasion on the average water contact angle of two coatings labelled A and B using cheese cloth (the least abrasive), is shown in Fig 2. Both coatings are non-fluorinated but differed in the concentration of nanoparticles.
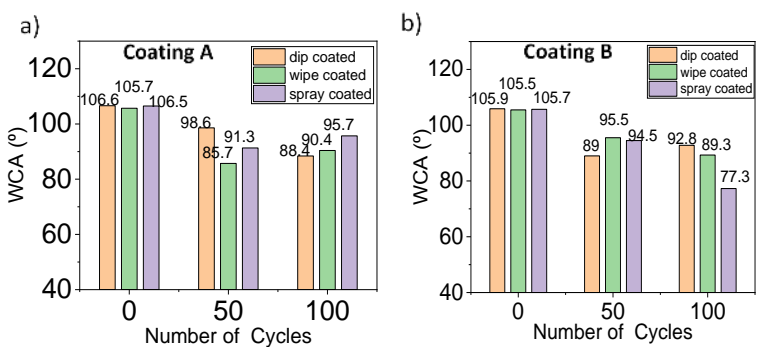

Fig. 2. a) Water contact angle of Coatings A and b) B by dip, wipe and spray coating methods before and after testing with cheesecloth.

Wear caused by using cheesecloth caused a drop in WCA after 50 cycles (Fig. 2a) for both coatings. However, the different methods of application affected the abrasion resistance. The degradation continues after 100 cycles with a small recovery in WCA for wipe and spray coating A. The dip coating
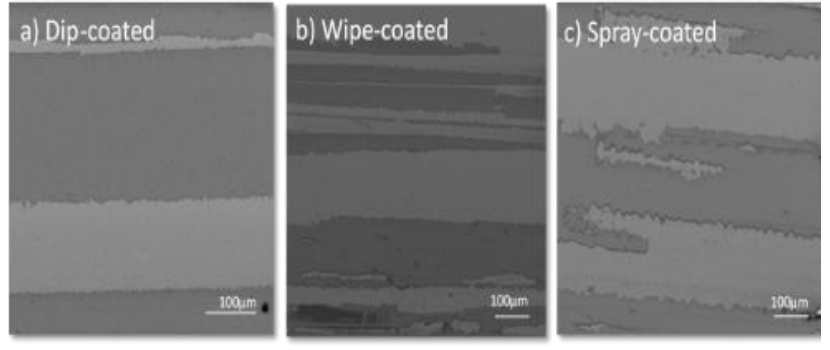

Fig. 3. a-c): SEM images of coating A after 100 cycles of abrasion with cheesecloth.

lost the hydrophobicity (cut off $90^{\circ}$ ) after 100 cycles. Whereas, for Coating B, Fig. 2b, only the sprayed coating recovers after 100 cycles although SEM images of these coatings show areas of coating delamination in Fig. 3a)-c). Preliminary results indicate low abrasion resistance of the coatings tested.

The use of four different abraders including felt pad, cheese cloth, CS-10 and CS-8 was investigated on coatings prepared using the same hydrophobic formulation. The abrasion was assessed by measuring their WCA and RoA as shown in Fig. 4.
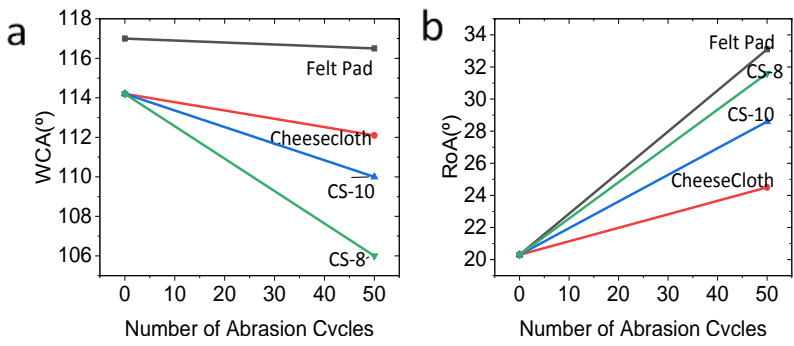

Fig. 4. a): Impact of abrasion on WCA and b) RoA of a fluorinated coating

Fig. 4a), b) shows a trend of decreasing WCA and increasing RoA due to the wear caused by the abraders. We note the most aggressive abrader was CS-8 and caused the most 
severe decline in WCA ( $8^{\circ}$ after 50 cycles) of the fluorinated, hydrophobic coating applied by spraying. However, the highest RoA was caused by the felt pad which may be linked to increased surface roughness. The cheese cloth and felt pad caused least $\left(\sim 2^{\circ}\right)$ decline in WCA after 50 cycles of abrasion.

Coatings remain hydrophobic since the WCA was above 90o, although the SEM images, Fig. 5 show deep scratches exposing the glass surface. The maximum increase in RoA was for felt pad, $63 \%$ from the initial RoA; $20.3^{\circ}$, followed by CS$8, \sim 51 \%$. The $20.7 \%$ increase in RoA for cheesecloth was the lowest. SEM images Fig. 5 (e-h) show the scratches produced by cheese cloth and felt pad abrasion.

Based on the observations from SEM and measurements of WCA and RoA the use of a softer cloth such as cheesecloth likely to prolong the functionality these anti-soiling coatings. Coupons exposed to CS-10 and CS-8 abraders show coating delamination. Although the WCAs for coupons exposed to felt pad and cheesecloth are degraded, scratches to the underlying glass did not occur.

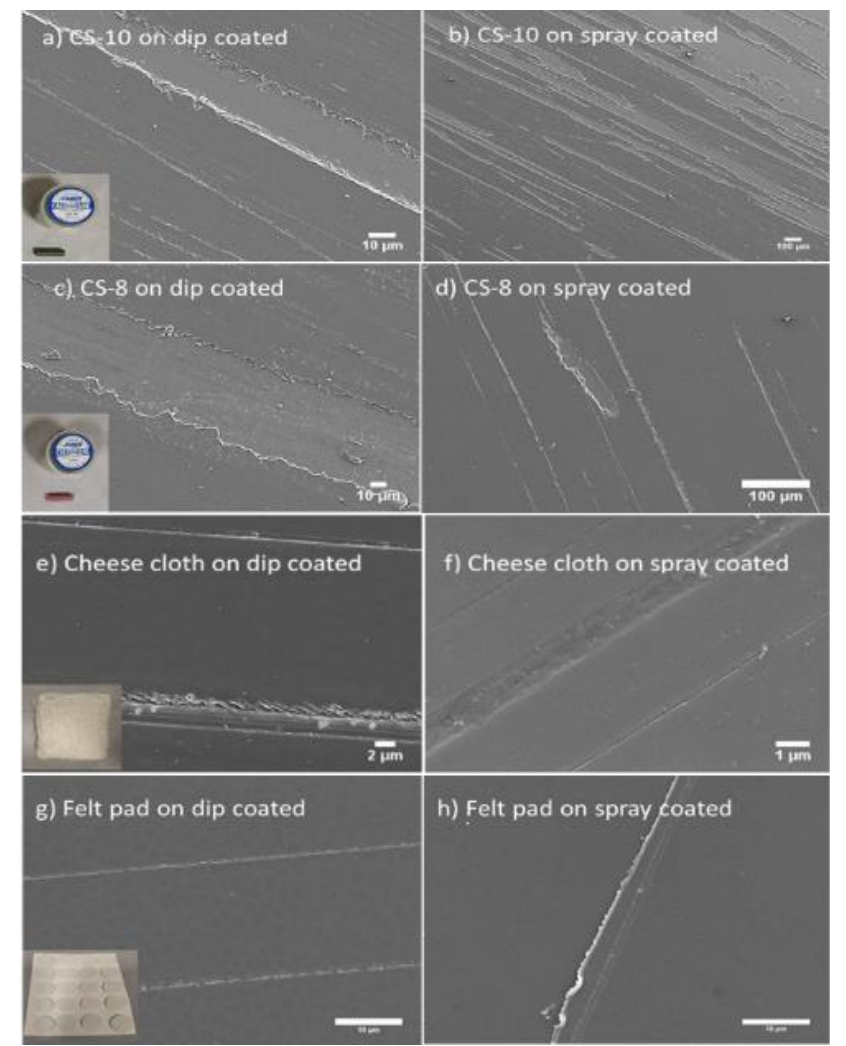

Fig. 5 (a-h): SEM images of dip and spray coated coupons after abrasion by CS-10 (a-b), CS-8 (c-d), Cheesecloth, (e- f) and Felt pad (g- h)

\section{B. Washability Tests}

Preliminary results for coating A are shown in Fig. 6. Higher WCAs were maintained with wild boar brush, nylon brush and sponge pads, whereas abrasive pad caused a significant damage to the coating. RoAs remained low for nylon brush and sponge pads although some structural damage was observed in the form of scratches.
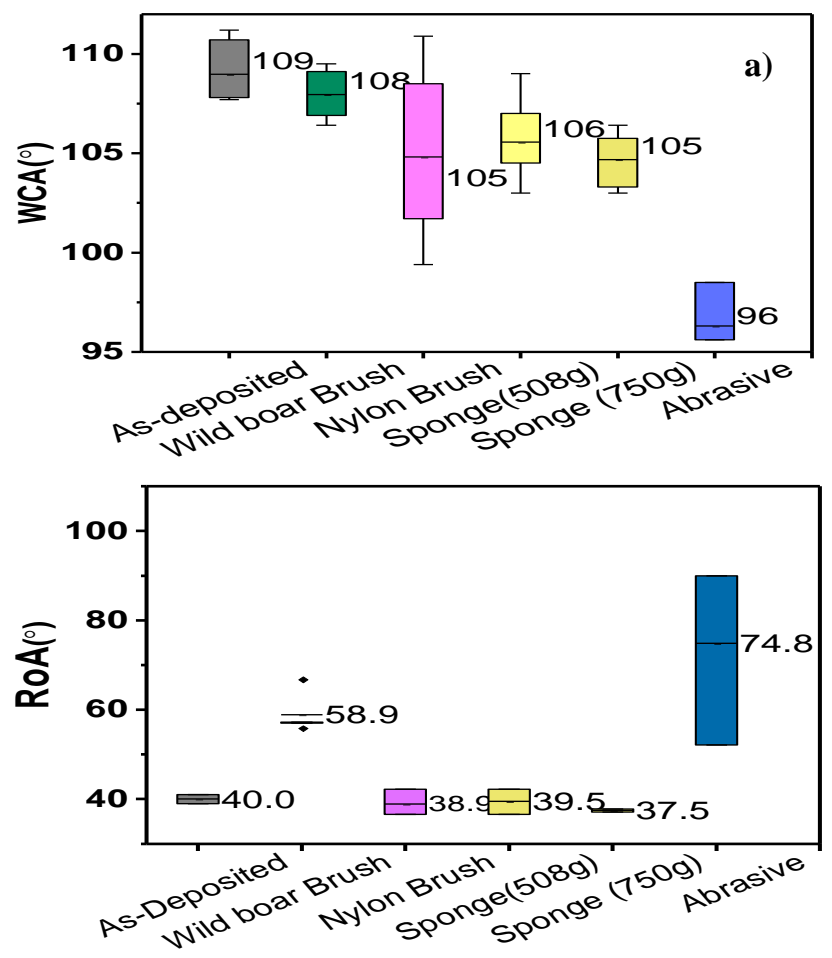

Fig. 6: a) Average WCAs and b) RoAs of anti-soiling coating A before and after washability testing with 5 tools

Tool 1, wild boar brush, sponge (508g) and Nylon brush caused about $\sim 1^{\circ}$ and $4^{\circ}$ decline in WCA after 50 cycles of abrasion.

The metallic 'abrasive pad' was the most aggressive tool and lowered the WCA to $\sim 96^{\circ}$ after 200 cycles. The coatings lost their hydrophobicity by using abrasive pad tool which is metallic in nature. However, interestingly, the average RoAs improved after performing the washability tests for tools except those of abrasive pad and wild boar brush.

SEM images in Fig. 7 show damage caused to the coating after performing tests. The micro-scale scratching justify the changes in hydrophobic characteristics.

Based on these results, the use of a softer material such as felt, sponge pad, wild boar and Nylon brushes for cleaning is likely to preserve coatings for a longer period. The use of water improved the ease of cleaning and reduced surface damage for all the coatings we tested. 


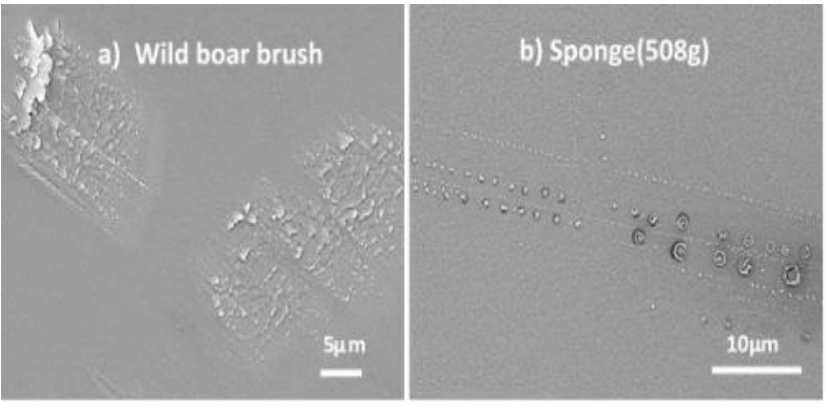

Fig. 7: a) SEM images of coating A after 200 cycles of abrasion with a) wild boar brush and b) Sponge (508g).

Box plots for coating B are shown in Fig. 8. Interestingly, the WCA values were like those for coating A. High RoA values indicate significant change in surface roughness, making it difficult for the droplets to roll across the surface. Hence, it can be asserted that use of abrasive pad can ruled out while performing such tests in future. Although the coatings differ in chemical composition, they have shown comparable results for

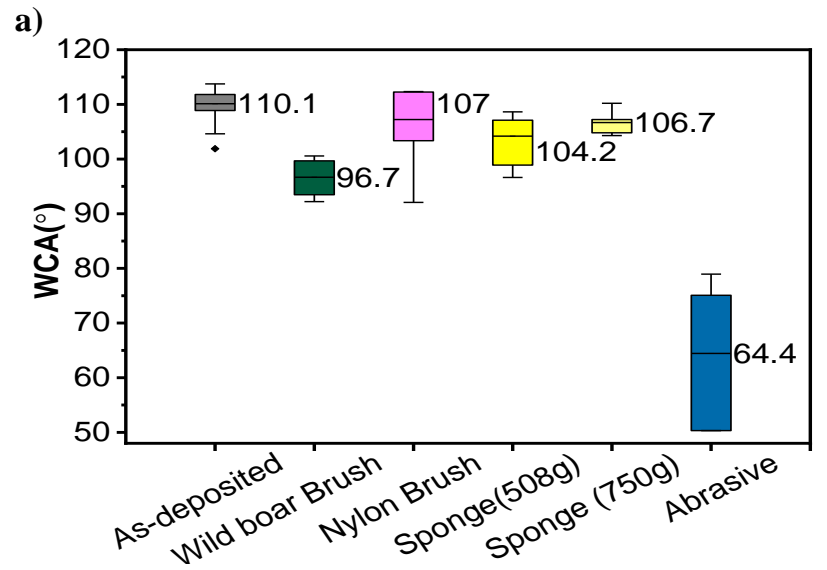

b)

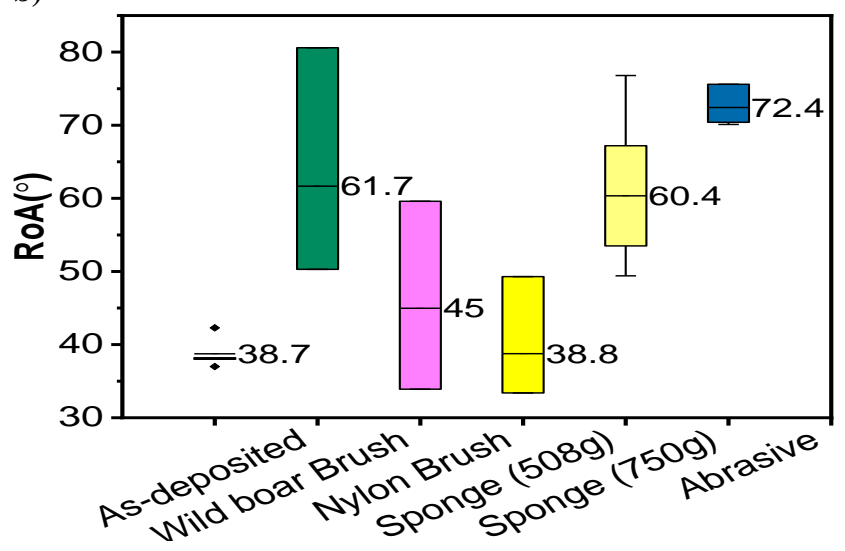

Fig. 8. a) Average WCAs and b) RoAs of anti-soiling coating B before and after washability testing with 5 tools.

washability tests SEM images in Fig. 9 and Fig. 7, i.e., scratching at micro scale. Abrasion resistance is important in maintaining high contact angles and therefore continue to possess their self-cleaning properties. Adhesion force

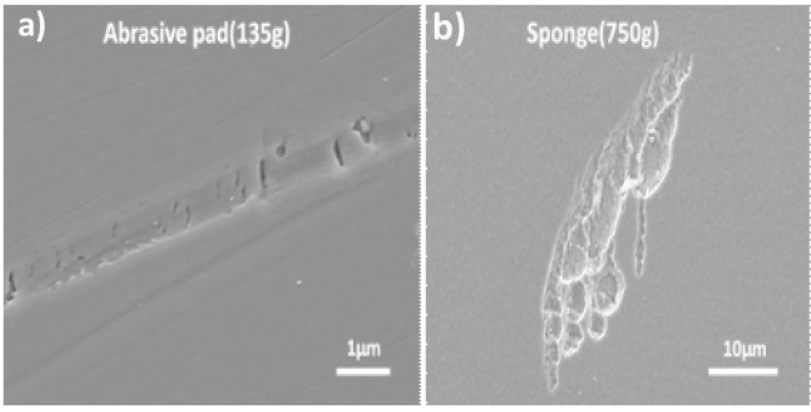

Fig. 9: a) SEM images of coating B after 200 cycles of abrasion with a) Abrasive pad and b) Sponge (750g).

measurements can confirm the results shown by SEM images, which will be reported in further work.

Comparison of these results with outdoor exposed coupons aided in facilitating the significance of these tests. A similar abrasion was observed (Fig. 10b)) when spray coating A was exposed outdoor for four weeks of between March $20^{\text {th }}, 2019$ and April $20^{\text {th }} 2019[1]$. The glass coupon with coating was cleaned of cemented dust particles using water and micro-fibre cloth. The adhered particles dragged across the surface whilst cleaning and caused abrasion.

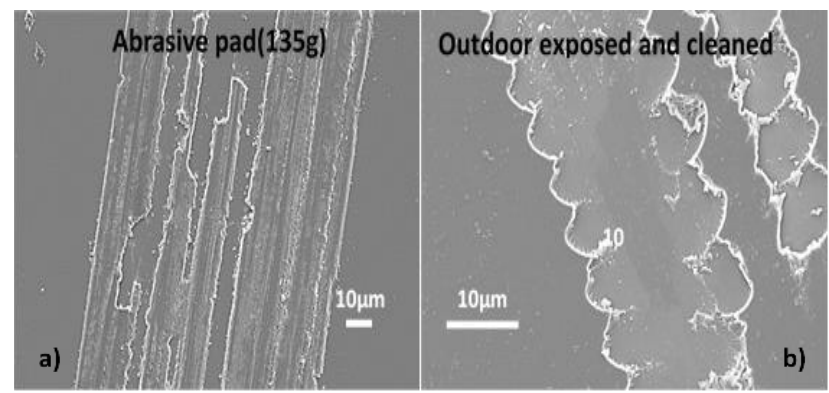

Fig. 10: a) SEM images of coating A after 200 cycles of abrasion with a) abrasive pad and b) with microfiber cloth

Morphologically, these scratches are comparable to those created by our 50 cycles of linear abrasion tests using CS-8 and CS-10 (Fig. 5(a-d) under 5N load and with sponge (fig. 9b), and abrasive pads with added load of 135g. Resistance to such abrasion is of high importance for any potential coating to maintain its hydrophobicity and self-cleaning properties. This could possibly be achieved by choosing a chemical composition that 'recovers' itself after such damage or limiting material removal during such abrasion exercises in the field.

\section{CONCLUSION}

Abrasion resistance tests were carried out on candidate hydrophobic coatings with different formulations, cured under different conditions and deposited by different methods. The tests show that coating hardness and adhesion to the glass substrate are important factors for the PV application. The tests do not directly simulate the abrasion induced during outdoor 
exposure, although, the preliminary results indicate that damage caused after cleaning an outdoor exposed coupon is similar to the abrasion caused by some of our tested tools. We suggest, that the choice of cleaning materials must be clearly specified to match the abrasion resistance of the coating used. Rigorous testing is also required to avoid large scale problems in the field.

\section{REFERENCES}

[1] G. C. Oehler et al., "Testing the durability of anti-soiling coatings for solar cover glass by outdoor exposure in Denmark," Energies, vol. 13, no. 2, 2020.

[2] S. F. Bukhari et al., "Development of a Hydrophobic , Anti-soiling coating for PV Module Cover Glass," 46th IEEE PVSC, 2019.

[3] G. Womack, K. Isbilir, F. Lisco, G. Durand, A. Taylor, and J. M. Walls, "The performance and durability of single-layer sol-gel anti-reflection coatings applied to solar module cover glass," Surf. Coatings Technol., vol. 358, pp. 76-83, 2019.

[4] D. C. Miller, M. T. Muller, and L. . Simpson, "Review of artificial abrasion test methods for PV module technology," no. August, p. 25, 2016.

[5] "BS EN 61215-1-3: 2017 BSI Standards Publication Terrestrial photovoltaic ( PV ) modules - Design qualification and type approval," 2017.

[6] Dansk Standard, "Glass in building - Coated glass - Part 3: Requirements and test methods for class C and D coatings," vol. DS/EN 1096, pp. 1-16, 2001.

[7] ISO, "Optics and photonics - Optical coatings, Part 4: Specific test methods," Iso 9211-4, 2012.

[8] I. Standard, "Iso 11998," vol. 1998, 1998.

[9] ASTM, "Standard Test Methods for Scrub Resistance of Wall Paints 1," ASTM Int., vol. 6, no. August 1996, pp. 6-9, 2015.

[10] R. Products, A. Coatings, R. Materials, and G. Electrode, "Standard Test Method for Scrub Resistance of Paints by Abrasion Weight Loss - ASTM D 4213 - 08," ASTM Int., vol. 96, no. Reapproved, pp. 1-5, 2008. 